Pacific Journal of Mathematics

SOME REMARKS ON THE CENTER OF THE UNIVERSAL
ENVELOPING ALGEBRA OF A CLASSICAL SIMPLE L

Michael A. Gauger 


\title{
SOME REMARKS ON THE CENTER OF THE UNIVERSAL ENVELOPING ALGEBRA OF A CLASSICAL SIMPLE LIE ALGEBRA
}

\author{
Michael A. Gauger
}

This paper is concerned with explicitly producing generating sets of the centers of the universal enveloping algebras of classical simple Lie algebras.

Let $L$ be a finite-dimensional simple Lie algebra over an algebraically closed field $K$ of characteristic zero, let $U$ be its universal enveloping algebra, and let $Z$ be the center of $U$. If $l$ is the dimension of a Cartan subalgebra $H$ of $L$, then it is known that $Z$ is a polynomial ring in $l$ independent variables. In this paper a set of $l$ algebraically independent generators of $Z$ is produced rather explicitly for the classical algebras of type $A, B, C, D$ by casewise considerations.

It is straightforward to show that generating $Z$ is equivalent to generating the $L$-invariants $I_{L}^{*}$ in the symmetric algebra $S_{L^{*}}$ of $L^{*}$. In addition, there is a homomorphism from $S_{L}$. onto $S_{H^{*}}$ which embeds $I_{L}^{*}$ into the Weyl-invariants $I_{W}$. Due to Chevalley this embedding is also a surjection. For the classical simple Lie algebras the action of the Weyl group $W$ on $S_{H^{*}}$ is describable in a sufficiently convenient fashion so as to permit easy construction of generators of $I_{W}$. It is shown here that certain generating sets of $I_{W}$ can be explicitly lifted back to $I_{L}^{*}$ via trace functions on the first fundamental representation of $L$. As a result of this construction of the generators of $I_{W}$ and the lifting process, the following well-known results are proven rather directly for the classical algebras:

1. $I_{L}^{*} \cong I_{W}$ (Chevalley), and

2. $Z$ and $I_{W}$ are polynomial rings in $l$ algebraically independent variables.

The center $Z$ of $U$ plays a fundamental role in the finitedimensional representation theory of $L$. Since any irreducible representation is determined up to isomorphism by its character, if $z_{1}, \cdots, z_{l}$ were generators of $Z$ and if $M$ and $N$ were non-isomorphic irreducible $L$-modules, then for some $i$ one must have $\left(z_{i}\right)_{M} \neq\left(z_{i}\right)_{N}$ (due to Schur's lemma they are scalars). The central element $\left(z_{i}-\left(z_{i}\right)_{N}\right) /\left(\left(z_{i}\right)_{M}-\left(z_{i}\right)_{N}\right)$ would act as one on $M$ and zero on $N$. For any list of pairwise non-isomorphic irreducible $L$-modules one could thus find a central element acting as one on one of them, and as zero on the rest. Such elements could be used to isolate the isotypic components in a reducible 
representation of $L$. Hence there is good reason to produce generators of $Z$ as explicitly as possible.

Section 1 is concerned with showing that generating $Z$ is equivalent to generating $I_{L}^{*}$ and leads up to $\S \S 2-5$ where the Chevalley isomorphism $I_{L}^{*} \cong I_{W}$ is proven by explicitly lifting generating sets of $I_{W}$ back to $I_{L}^{*}$.

1. Generation of $Z$. There are well known actions of $L$ on the symmetric algebras $S_{L}$ and $S_{L}$. by graded derivations extending the adjoint representation of $L$ and its contragredient, and if $W$ is the Weyl group of $L$ with respect to the Cartan subalgebra $H$, it acts on $S_{H}$. by graded automorphisms. The standard symmetrization map $\eta: S_{L} \rightarrow U$ given by $\left(x_{1} \cdots x_{r}\right)^{\eta}=(1 / r !) \Sigma_{\alpha \in S_{r}} x_{\alpha(1)} \cdots x_{\alpha(r)}$ for a monomial of degree $r$ in $S_{L}$, induces a linear isomorphism between the $L$-invariants $I_{L}$ in $S_{L}$ and the $L$-invariants $Z$ in $U$ since it is an $L$-module isomorphism. While this induced map between $I_{L}$ and $Z$ is not an algebra isomorphism it is known to have the following redeeming qualities:

LEMMA. Suppose $S$ is a finite set of homogeneous invariant elements in $S_{L}$ generating $I_{L}$. Then $S^{\eta}$ generates $Z$, and if $S$ is algebraically independent so is $S^{\eta}$.

Proof. Let $U$ have its usual filtration and let $U_{p}$ be the subspace of all elements of filter less than or equal to $p$. Observe that due to the Poincaré-Birkhoff-Witt theorem, if $x_{1}, \cdots, x_{r}$ are homogeneous elements of $S_{L}$ of degrees $d_{1}, \cdots, d_{r}$ and $d=\Sigma_{i} d_{t}$, then $\left(x_{1} \cdots x_{r}\right)^{\eta}=x_{1}^{\eta} \cdots x_{r}^{\eta}+t$ where $t$ is in $U_{d-1}$.

(i) Since $L$ acts by graded derivations, $I_{L}$ is homogeneous. Recalling that $\eta$ induces a linear isomorphism between $I_{L}$ and $Z$, proceed by induction on the filter of a central element to show it is in the subalgebra of $U$ generated by $S^{\eta}$. Let $S=\left\{x_{1}, \cdots, x_{r}\right\}$. Now $\eta$ takes constants to constants so it suffices to check the induction step. Every element in $Z$ is a linear combination of images of homogeneous elements in $I_{L}$, so it suffices to show that if $x_{i_{1}} \cdots x_{i_{k}}$ is a monomial in $I_{L}$ then $\left(x_{i_{1}} \cdots x_{l_{k}}\right)^{\eta}$ is in the subalgebra generated by $S^{\eta}$. The remarks in the first paragraph complete the proof.

(ii) Set $y_{t}=x_{l}^{\eta}$. Suppose the $y_{t}$ are algebraically dependent and let $p$ be a nonzero polynomial in $K\left[Y_{1}, \cdots, Y_{r}\right]$ such that $p\left(y_{1}, \cdots, y_{r}\right)=$ 0 . Write $p=q+t$ where $q$ is the homogeneous part of $p$ of highest total degree $d$. Since $\eta$ takes $q\left(x_{1}, \cdots, x_{r}\right)$ onto $q\left(y_{1}, \cdots, y_{r}\right)$ plus an element $u\left(y_{1}, \cdots, y_{r}\right)$ whose filter is less than $d$, there is a polynomial $h$ of degree less than $d$ such that $\eta$ takes $h\left(x_{1}, \cdots, x_{r}\right)$ onto $t\left(y_{1}, \cdots, y_{r}\right)-$ $u\left(y_{1}, \cdots, y_{r}\right)$. Since $\eta$ is an isomorphism $(q+h)\left(x_{1}, \cdots, x_{r}\right)=0$. This contradicts the independence of the $x_{i}$ since $q+h \neq 0$.

Since the Killing form of $L$ is nondegenerate there is an induced 
isomorphism between $L$ and $L^{*}$ which extends to an $L$-module algebra isomorphism between $S_{L}$ and $S_{L^{*}}$. Hence there is an induced algebra isomorphism between $I_{L}$ and $I_{L}^{*}$. Viewing $S_{L}$, as the ring of polynomial functions on $L$, one gets by restriction to $H$ an epimorphism $\rho: S_{L^{*}} \rightarrow S_{H^{*}}$ which injects $I_{L}^{*}$ into $I_{W}([2], 126)$. The remainder is concerned with producing algebraically independent generating sets of $I_{W}$ and exhibiting how they lift back to $I_{L}^{*}$. Chevalley's isomorphism $\left(I_{L}^{*} \cong I_{W}\right)$ is thus proven as well as the theorems that $\mathbb{Z}$ and $I_{W}$ are polynomial rings in $l$ independent variables.

2. Simple algebras of type $A$. Let $L$ be simple of type $A_{1}$. View $L$ as the Lie algebra of trace zero endomorphisms of $V=K^{l+1}$, and identify $L$ with its matrices with respect to standard basis vectors $e_{1}, \cdots, e_{l+1}$. Let $H$ be the Cartan subalgebra of diagonal matrices of trace zero and let $\epsilon_{1}, \cdots, \epsilon_{l+1}$ be functionals on $H$ given by $\left(e_{i}\right) h=\epsilon_{i}(h) e_{i}$ for $h$ in $H$. Then the $\epsilon_{j}$ generate $H^{*}, \Sigma_{j} \epsilon_{j}=0$, and $W$ acts as the symmetric group on the $\epsilon_{\jmath}$. ([3], 136 and [1] 205-207, 250-251). Let $A$ be an $l+1$-dimensional auxiliary space with basis $\bar{\epsilon}_{1}, \cdots, \bar{\epsilon}_{l+1}$ on which $W$ acts as the symmetric group. There is a $W$-epimorphism $A \rightarrow H^{*}$ taking $\bar{\epsilon}_{i}$ to $\epsilon_{l}$ which extends to a $W$ epimorpism $S_{A} \rightarrow S_{H^{*}}$. Hence there is an induced epimorphism $\bar{I}_{W} \rightarrow I_{W}$ where $\bar{I}_{W}$ is the set of $W$-invariants in $S_{A}$. Now $\bar{I}_{W}$ is generated by the (algebraically independent) elementary symmetric functions in $\bar{\epsilon}_{1}, \cdots, \bar{\epsilon}_{l+1}$. The kernel of $\bar{I}_{W} \rightarrow I_{W}$ is easily seen to be generated by $\Sigma_{j} \bar{\epsilon}_{j}$, thus $I_{W}$ is generated by the algebraically independent elementary symmetric functions $s_{2}, \cdots, s_{l+1}$ in $\epsilon_{1}, \cdots, \epsilon_{l+1}$ - the analysis being identical to the situation $K\left[X_{1}, \cdots, X_{l+1}\right] \rightarrow K\left[X_{1}, \cdots, X_{l}\right]$ where $X_{l+1}$ goes to zero. Unfortunately the symmetric functions do not lift easily. Due to Newton's identities, however, $I_{W}=K\left[p_{2}, \cdots, p_{l+1}\right]$ where $p_{i}=$ $\epsilon_{1}^{i}+\cdots+\epsilon_{l+1}^{i}$ and the $p_{i}$ do lift easily. They are algebraically independent since they generate a ring known to have transcendence degree equal to $l$. Now let $F_{l}$ in $I_{L}^{*}$ be given by $F_{i}(x)=\operatorname{tr}\left(x_{V}\right)^{i}$. Then $F_{i}^{\boldsymbol{p}}=p_{i}$ and $\rho: I_{L}^{*} \rightarrow I_{W}$ is surjective. Under the isomorphisms $Z \simeq I_{L} \simeq I_{L}^{*}$ the element $z_{k}$ of $Z$ corresponding to $F_{k}$ is given by

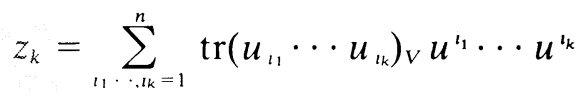

where $\left\{u_{i}\right\},\left\{u^{\prime}\right\}$ are dual bases of $L$ with respect to its Killing form. By Lemma 1 and the discussion in $\S 1 Z=K\left[z_{2}, \cdots, z_{l+1}\right]$ and the $z_{k}$ are algebraically independent.

3. Simple algebras of type $B$. Let $L$ be a simple algebra of type $B_{l}$. Let $V$ be a $(2 l+1)$-dimensional space with basis $e_{1}, \cdots, e_{2 l+1}$, 
and define a non-degenerate symmetric form on $V$ by $B\left(e_{1}, e_{1}\right)=1=$ $B\left(e_{l}, e_{t+l}\right)=B\left(e_{t+l}, e_{l}\right) i=2, \cdots, l+1$ and $B\left(e_{j}, e_{k}\right)=0$ otherwise. View $L$ as the Lie algebra of all endomorphisms of $V$ which are skew with respect to this form and identify $L$ with its matrices with respect to the $e_{1}$. Let $H$ be the Cartan subalgebra of diagonal matrices in $L$, and let $\epsilon_{1}, \cdots, \epsilon_{l}$ be functionals on $H$ given by $\left(e_{l}\right) h=\epsilon_{i}(h) e_{i}$ for $h$ in $H$ ([3], 138). Then $\left\{\epsilon_{k}\right\}_{k}$ is a basis of $H^{*}$ and $W$ is the semidirect product of the symmetric group $S_{l}$ on $\epsilon_{l}, \cdots, \epsilon_{l}$ with $(\mathbf{Z} / 2 \mathbf{Z})^{l}$ acting by $\epsilon_{i} \rightarrow( \pm 1)_{l} \epsilon_{i}$. Thus $I_{W}$ consists of symmetric functions in $\epsilon_{1}^{2}, \cdots, \epsilon_{l}^{2}$ ([2], 202 and 252). By Newton's identities $I_{W}=k\left[p_{1}, \cdots, p_{l}\right]$ where $p_{i}=\epsilon_{1}^{2 i}+\cdots+\epsilon_{l}^{2 i}$. Since $\epsilon_{i}^{2}, \cdots, \epsilon_{i}^{2}$ are algebraically independent, so are the $p_{i}$. Let $F_{i}$ in $I_{L}^{*}$ be given by $F_{l}(x)=\operatorname{tr}\left(x_{V}\right)^{2 i}$. Then $F_{\imath}^{\rho}=2 p_{t}$ and $\rho$ is onto. $Z=$ $K\left[z_{2}, z_{4}, \cdots, z_{2 l}\right]$ where the $z_{2 k}$ are as in (1).

4. Simple algebras of type C. Let $L$ be simple of type $C_{l}$. Let $V$ be a $2 l$-dimensional space with basis $e_{1}, \cdots, e_{2 l}$, and define a nondegenerate skew form on $V$ by $B\left(e_{i}, e_{i+l}\right)=1=-B\left(e_{i+l}, e_{i}\right) i=$ $1, \cdots, l$ and $B\left(e_{f}, e_{k}\right)=0$ otherwise. View $L$ as the Lie algebra of endomorphisms which are skew with respect to this form, and identify $L$ with its matrices with respect to the $e_{i}$. Let $H$ be the Cartan subalgebra of diagonal matrices in $L$, and let $\epsilon_{1}, \cdots, \epsilon_{l}$ be functionals on $H$ given by $\left(e_{l}\right) h=\epsilon_{l}(h) e_{t}$ when $h$ is $H([3], 139)$. Then $\epsilon_{1}, \cdots, \epsilon_{l}$ is a basis of $H^{*}$, $W$ acts just as in the preceding case, and $I_{W}$ consists of symmetric functions in $\epsilon_{1}^{2}, \cdots, \epsilon_{l}^{2}([2], 204 \& 254)$. As before one sees $\rho$ is onto and $Z=K\left[z_{2}, \cdots, z_{2 l}\right]$ where $z_{2 k}$ is as in (1).

5. Simple algebras of type $D$. Let $L$ be simple of type $D_{l}$. Let $V$ be a $2 l$-dimensional space with basis $e_{1}, \cdots, e_{2 l}$, and define a nondegenerate symmetric form on $V$ by $B\left(e_{i}, e_{i+l}\right)=1=B\left(e_{i+l}, e_{l}\right) i=$ $1, \cdots, l$ and $B\left(e_{l}, e_{k}\right)=0$ otherwise. View $L$ as the Lie algebra of endomorphisms of $V$ which are skew with respect to this form and identify $L$ with it matrices with respect to the $e_{j}$. Let $H$ be the Cartan subalgebra of diagonal matrices in $L$ and let $\epsilon_{1}, \cdots, \epsilon_{l}$ be functionals on $H$ given by $\left(e_{l}\right) h=\epsilon_{l}(h) e_{t}$ when $h$ is in $H([3], 140)$. Then $\epsilon_{1}, \cdots, \epsilon_{l}$ is a basis of $H^{*}$ and $W$ is the semi-direct product of the symmetric group $S_{l}$ acting as before with $(\mathbf{Z} / 2 \mathbf{Z})^{l-1}$ acting by $\epsilon_{i} \rightarrow( \pm 1)_{i} \epsilon_{i}$ where $\Pi_{i}( \pm 1)_{i}=1$ ([2], 208 and 256). Thus $I_{W}$ consists of polynomials in the elementary symmetric functions in $\epsilon_{1}^{2}, \cdots, \epsilon_{l}^{2}$ and the function $\epsilon_{1} \cdots \epsilon_{l}$. Let $s_{k}$ be the $k$ th elementary symmetric function in the $\epsilon_{i}^{2}$ and let $t=\epsilon_{1} \cdots \epsilon_{l}$. Since $s_{l}=t^{2}$, one has $I_{W}=K\left[s_{1}, \cdots, s_{l-1}, t\right]$. If $s_{1}, \cdots, s_{l-1}, t$ were algebraically dependent, by an even-odd degree argument there would be a relation in which every monomial has $t$ to an even power, or every monomial has $t$ to an odd power. If the relation is of the second type multiply it by $t$ to make it of the first type. But a relation of the first type is impossible 
since the elementary symmetric function in the $\epsilon_{i}^{2}$ are algebraically independent. Thus $I_{W}$ is a polynomial ring in $l$ independent variables. By Newton's identities $I_{W}=K\left[p_{1}, \cdots, p_{l-1}, t\right]$ where $p_{i}=$ $\epsilon_{1}^{2 i}+\cdots+\epsilon_{l}^{2 i}$. These generators are also algebraically independent since there are $l$ of them in a ring known to have transcendence degree equal to $l$. As before $2 p$, lifts back to $I_{L}^{*}$ as $\operatorname{tr}()_{v}^{2 i}$, and it is easy to check that $t=\epsilon_{1} \cdots \epsilon_{l}$ lifts back to $I_{L}^{*}$ as $p f()_{V}$ - the pfaffian. Thus $\rho$ is onto and $Z=K\left[z_{2}, z_{4}, \cdots, z_{2 l-2}, w\right]$ where the $z_{2 k}$ are as in (1) and $w$ corresponds to $p f()_{V}$ under $Z \simeq I_{L} \simeq I_{L}^{*}$.

Remark. Dual bases of $L$ with respect to its Killing Form can be explicitly constructed ([3], 246; $h^{i}=h_{\lambda_{i}}$ where the $\lambda_{i}$ are the fundamental weights). According to part VI of Planche I-IV ([1], 250-258) the coefficients $q_{i j}$ of the equations $\lambda_{l}=\sum_{j} q_{i j} \alpha_{j}\left(\alpha_{1}, \cdots, \alpha_{l}\right.$ a simple root system) are known, thus enabling one to express $h^{i}$ explicitly as a Q-linear combination of the $h_{i}$.

\section{REFERENCES}

1. N. Bourbaki, Elements de Mathématique, XXXIV Groupes et algèbres de Lie. Ch. 6: Systèmes de racines, Actualités Sci. Indust., no. 1337, Hermann, Paris, 1968.

2. J. Humphreys, Introduction to Lie Algebras and Representation Theory, Springer-Verlag, N.Y. 1972.

3. N. Jacobson, Lie Algebras, Interscience Tracts in Pure and Applied Math., no. 10, Interscience, New York, 1962.

Received May 15, 1974. The research presented here was supported in part by the National Science Foundation under grant GP-43814. 




\section{Pacific Journal of Mathematics}

\section{Vol. 62, No. $1 \quad$ January, 1976}

Mieczyslaw Altman, Contractor directions, directional contractors and

directional contractions for solving equations . .................. 1

Michael Peter Anderson, Subgroups of finite index in profinite groups .........

Zvi Arad, Abelian and nilpotent subgroups of maximal order of groups of odd order

John David Baildon and Ruth Silverman, On starshaped sets and Helly-type theorems ..........................................

John W. Baker and R. C. Lacher, Some mappings which do not admit an

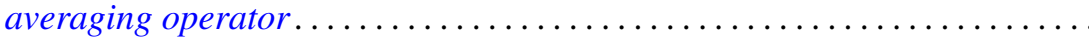

Joseph Barback, Composite numbers and prime regressive isols . . . . . . . . . .

David M. Boyd, Composition operators on $H^{p}(A) \ldots \ldots \ldots \ldots \ldots \ldots \ldots$

Maurice Chacron, Co-radical extension of PI rings . . . . . . . . . . . . .

Fred D. Crary, Some new engulfing theorems . . . . . . . . . . . . . . .

Victor Dannon and Dany Leviatan, A representation theorem for convolution transform with determining function in $L^{p} \ldots \ldots \ldots \ldots \ldots \ldots \ldots \ldots \ldots \ldots \ldots \ldots \ldots \ldots$

Mahlon M. Day, Lumpy subsets in left-amenable locally compact

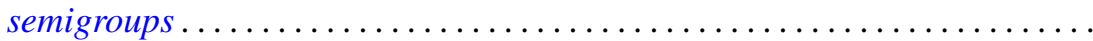

Michael A. Gauger, Some remarks on the center of the universal enveloping algebra of a classical simple Lie algebra . .

David K. Haley, Equational compactness and compact topologies in rings

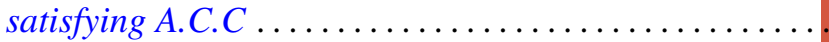

Raymond Heitmann, Generating ideals in Prüfer domains .

Gerald Norman Hile, Entire solutions of linear elliptic equations with

Laplacian principal part. .

Richard Oscar Hill, Moore-Postnikov towers for fibrations in which $\pi_{1}$ (fiber) is non-abelian

John Rast Hubbard, Approximation of compact homogeneous maps . .

Russell L. Merris, Relations among generalized matrix functions . .

V. S. Ramamurthi and Edgar Andrews Rutter, On cotorsion radicals ...

Ralph Tyrrell Rockafellar and Roger Jean-Baptiste Robert Wets, Stochastic

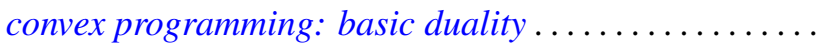

Alban J. Roques, Local evolution systems in general Banach spaces ..

I. Bert Russak, An indirect sufficiency proof for problems with bounded state variables.

Richard Alexander Sanerib, Jr., Ultrafilters and the basis property. .

H. A. Seid, The decomposition of multiplication operators on $L_{p}$-spaces . .

Franklin D. Tall, The density topology .................. 